\title{
Periodic solutions and global indeterminacy in a model of sustainable tourism
}

\section{Giovanni Bella ${ }^{1}$}

\begin{abstract}
The impact of tourism on economic growth and environmental degradation is one of the most relevant debated issues. Despite the huge strand of empirical literature on the topic, a formalized theoretical investigation of the link between tourism and sustainable economic growth is unfortunately still lacking. To this end, and in line with the literature on the tourist lifecycle hypothesis, we present an endogenous growth model to study the impact of tourism activities and natural resource use on the long run steady state. The aim is to use the principles of bifurcation theory to gain hints on the global properties of the equilibrium, and show the existence of irregular patterns, either indeterminate or chaotic, which possibly suggest the emergence of a (low growth) poverty-environment trapping region.
\end{abstract}

Keywords: Sustainable tourism; Complex dynamics; Economic growth; Poverty trap.

To cite this article: Bella, G. (2017). Periodic solutions and global indeterminacy in a model of sustainable tourism. Journal of Tourism Theory and Research, 3(2), 89-97. DOI: 10.24288/jttr.314591

1'bella@unica.it 


\section{Introduction}

Tourism economics is characterized by an explosive growing interest. Unfortunately, formalized theoretical studies are still lacking in this field to explore the complex relationship existing between tourism and a sustainable economic growth (see, Sachs and Warner, 2001).

Nowadays economies basically share the common view that production of goods and services, especially for firms operating in the tourist sector, highly depend on natural resource overuse, and no growth is therefore possible without this input. Therefore, trying to understand the way an economy can grow along an optimal equilibrium path, without sacrificing the available natural resources, is one of the most debated and intriguing social claims, and is in line with the socalled tourism-led-growth hypothesis (Brau et al., 2007; Nowak et al., 2007; Baggio, 2008; Brida et al., 2008; Katircioglu, 2009; Bornhost et al., 2010; Schubert et al., 2010).

Interestingly, once natural resource exploitation is taken into account, attention can be immediately devoted to understand whether tourism might affect the long run dynamics towards a stable equilibrium, or if undesired indeterminacy problems may eventually arise. Moreover, the rise of indeterminacy in presence of an overuse of natural resources could be the major cause for the emergence of a vicious povertyenvironment trap, where public policies might not be able to avoid a non sustainable use of natural resources (see, for example, Finco, 2009).

A wide strand of literature has focused on the conditions for the emergence of multiple equilibrium trajectories in the vicinity of the steady state, but only very few attempts have been made to study the conditions for global indeterminacy and possible chaotic solutions to occur outside such small neighborhood of the steady state. In this case, despite the initial conditions or other economic fundamentals, the agents' decisions could locate the economy in a path which is not corresponding to the lowest exploitation level of natural resources (see, for example, Mattana and Venturi, 1999; Benhabib et al., 2001; Bella and Mattana, 2014).

In line with this strand of literature, we propose an endogenous growth model to study the impact of tourism activities and natural resource use on the long run steady state. The aim is to use the principles of bifurcation theory to gain hints on the global properties of the equilibrium, and show the existence of irregular patterns due to a sensitive dependence of our economy on the initial conditions. In particular, we study the presence of closed orbits, to detect the rise of economic fluctuations and periodic solutions around the steady state, which are empirically confirmed by the literature on the tourist life-cycle hypothesis (Butler, 1980). The problem is to understand the conditions under which these orbits are attracting, with equilibrium trajectories being captured by the orbit itself, that finally becomes a (indeterminate or maybe chaotic) limit set, which possibly suggests the emergence of a (low growth) poverty-environment trapping region.

The rest of the paper is organized as follows. First, we present the model, derive the steady state conditions, and study the local dynamics. Second, we characterize the parametric space where periodic solutions emerge, and the equilibrium becomes indeterminate, and use the Andronov-Hopf bifurcation theorem to study the global properties of the steady state. Lastly, a final section concludes, and a subsequent Appendix provides all the necessary proofs.

\section{The model}

We consider an optimal control problem where a representative agents aims at maximizing the lifetime utility, $U$, subject to the constraints on the accumulation of both physical capital, $k$, and the stock of available natural resources, $E$, given their positive initial values $k(0)=k_{0}>0$ and $E(0)=E_{0}>0$.

Let assume a standard CIES utility function, i.e. $U=\frac{c^{1-\sigma}-1}{1-\sigma}$, where $c$ is per capita consumption, and $\sigma$ is the inverse of the intertemporal elasticity of substitution. Assume also that the level of investment in physical capital is given by the usual functional form $\dot{k}=y-c$, where output $y$ is produced according to the function

$$
y=A k^{\alpha} Q^{1-\alpha}
$$

where $\alpha \in[0,1]$ is the share of physical capital, $A$ measures the stock of existing technology, with 
physical capital, $k$, entering as an input along with the total amount of tourism services, $Q$. The latter is additionally specified, as depending on the amount of public spending used to promote the tourism sector, $g$, and the available amount of natural resources, $E$, in a Cobb-Douglas evolutionary law

$Q=g^{\beta} E^{1-\beta}$

being $\beta$ a standard elasticity parameter.

Additionally, we set the dynamic evolution of the environmental sector as represented by $\dot{E}=N(E)-Z(Q)$, which is negatively affected by the extractive use of natural resources employed in the production of tourism services, $Z(Q)$, whereas $N(E)$ determines the speed at which nature regenerates. To simplify the analysis, we propose a linear representation of these functions, namely: $N(E)=\delta E$ and $Z(Q)=\theta Q$, where $\delta$ and $\theta$ denote constant parameters of scale (see also, Musu, 1995; Rosendahl, 1996; Cole, 2009).

Hence, under a constant time preference rate, $\rho$, the maximization problem explicitly becomes

$$
\operatorname{Max}_{c(t)} \int_{0}^{\infty} \frac{c^{1-\sigma}-1}{1-\sigma} e^{-\rho t} d t
$$

s.t.

$\dot{k}=A k^{\alpha} Q^{1-\alpha}-c$

$\dot{E}=\delta\left(E-g^{\beta} E^{1-\beta}\right)$

with an associated current value Hamiltonian given by

$H_{C}=\frac{c^{1-\sigma}-1}{1-\sigma}+\lambda\left[A k^{\alpha} g^{\beta(1-\alpha)} E^{(1-\beta)(1-\alpha)}-c\right]+\mu \delta\left(E-g^{\beta} E^{1-\beta}\right)$

where $\lambda$ and $\mu$ represent the shadow prices of physical capital and natural resources, respectively.

Solution to this optimal control problem implies the following first order necessary conditions:

$c^{-\sigma}=\lambda$

$\lambda(1-\alpha) A k^{\alpha}=\mu \delta g^{\beta \alpha} E^{(1-\beta) \alpha}$ accompanied by the equation of motion for each costate variable, that can be derived with a bit of mathematical manipulation as

$\frac{\dot{\lambda}}{\lambda}=\rho-\alpha A k^{\alpha-1} g^{\beta(1-\alpha)} E^{(1-\beta)(1-\alpha)}$

$\frac{\dot{\mu}}{\mu}=\rho-(1-\beta) \frac{\lambda}{\mu}(1-\alpha) A k^{\alpha} g^{\beta(1-\alpha)} E^{(1-\beta)(1-\alpha)-1}-\delta\left[1-(1-\beta) g^{\beta} E^{-\beta}\right]$

and the transversality condition

$\lim _{t \rightarrow \infty} e^{-\rho t}\left[\lambda_{t} k_{t}+\mu_{t} E_{t}\right]=0$

that jointly constitute the canonical system. Since both Arrow's sufficiency theorem and the transversality condition hold, the problem is therefore bounded and concave.

\subsection{The reduced model}

The standard procedure is conducted in this section to study the transitional dynamics of Problem $(P)$.

Proposition 1 The maximum principle associated with the decentralized optimization problem $(P)$ implies the following four-dimensional system of first order differential equations

$\frac{\dot{k}}{k}=A k^{\alpha-1} g^{\beta(1-\alpha)} E^{(1-\beta)(1-\alpha)}-\frac{c}{k}$

$\frac{\dot{E}}{E}=\delta-\theta g^{\beta} E^{-\beta}$

$\frac{\dot{c}}{c}=-\frac{\rho}{\sigma}+\frac{\alpha}{\sigma} A k^{\alpha-1} g^{\beta(1-\alpha)} E^{(1-\beta)(1-\alpha)}$

$\frac{\dot{g}}{g}=-\frac{x}{\beta}+\frac{\delta(1-\alpha+\alpha \beta)}{\beta \alpha}+\frac{\delta}{\beta}(1-\beta) g^{\beta} E^{-\beta}$

Proof. See the Appendix.

Lemma 1 System $S$ can be easily reduced to 


$$
\begin{aligned}
& \dot{x}=\left\{-\frac{\rho}{\sigma}+\left(\frac{\alpha-\sigma}{\sigma}\right) A q^{\alpha-1} z^{(1-\alpha)}+x\right\} x \\
& \dot{q}=\left\{-\delta+A q^{\alpha-1} z^{(1-\alpha)}-x+\theta z\right\} q \\
& \dot{z}=\left\{\frac{\delta(1-\alpha)}{\alpha}-x+\theta z\right\} z
\end{aligned}
$$

by means of the convenient variable substitutions: $x=\frac{c}{k}, q=\frac{k}{E}$, and $z=\left(\frac{g}{E}\right)^{\beta}$.

Proof. See the Appendix.

Remark 1 The steady state is a triplet $\left(x^{*}, q^{*}, z^{*}\right)$ which solves the reduced system $(R)$

$$
\begin{aligned}
& x^{*}=\frac{\delta(\sigma-\alpha)+\alpha \rho}{\alpha \sigma} \\
& A q^{*(\alpha-1)} z^{*(1-\alpha)}=\frac{\delta}{\alpha} \\
& z^{*}=\frac{\rho-\delta(1-\sigma)}{\theta \sigma}
\end{aligned}
$$

Since the Jacobian matrix associated with $(R)$ is

$$
\mathbf{J}=\left[\begin{array}{ccc}
x^{*} & \frac{\delta(\alpha-1)}{\alpha}\left(\frac{\alpha-\sigma}{\sigma}\right) \frac{x^{*}}{q^{*}} & \frac{\delta(1-\alpha)}{\alpha}\left(\frac{\alpha-\sigma}{\sigma}\right) \frac{x^{*}}{z^{*}} \\
q^{*} & \frac{\delta}{\alpha}(\alpha-1) & \theta q^{*}+\frac{\delta(1-\alpha)}{\alpha} \frac{q^{*}}{z^{*}} \\
-z^{*} & 0 & \theta z^{*}
\end{array}\right]
$$

Let

$\operatorname{det}(\lambda \mathbf{I}-\mathbf{J})=\lambda^{3}-\operatorname{tr}(\mathbf{J}) \lambda^{2}+B(\mathbf{J}) \lambda-\operatorname{Det}(\mathbf{J}) \quad$ be the characteristic polynomial of $\mathbf{J}$, where $\mathbf{I}$ is the identity matrix and $\operatorname{tr}(\mathbf{J}), B(\mathbf{J})$, and $\operatorname{Det}(\mathbf{J})$, are Trace, Sum of Principal Minors of order two, and Determinant associated with $\mathbf{J}$, respectively. Algebraic computation gives

$$
\begin{aligned}
& \operatorname{tr}(\mathbf{J})=2 \theta z^{*} \\
& \operatorname{Det}(\mathbf{J})=\frac{\delta \theta(1-\alpha)(2 \alpha-3 \sigma)}{\alpha \sigma} x^{*} z^{*} \\
& B(\mathbf{J})=\theta z^{*} x^{*}-(1-\alpha) \frac{\delta}{\alpha}\left[x^{*}+\theta z^{*}\right]
\end{aligned}
$$

Conditions (7.i) may serve us to characterize the behavior of the equilibrium trajectories wondering around the steady state. Unfortunately, this is not an easy task, when dealing with complicate nonlinear functions. To this end, we provide a set of conditions necessary to facilitate the study of both local and global dynamics.

\subsection{Periodic solutions}

Let us first study the possibility that periodic solutions do emerge, which is a first way to signal that the interior steady state can be indeterminate.

In detail, we apply the neat Andronov-Hopf bifurcation theorem to verify that there exists a parameter value at which a structure of closed orbits exists around the steady state solution. We choose $\sigma$ as the appropriate bifurcation parameter. To prove this, we need to check the following expression

$G(\sigma)=\frac{\rho-\delta(1-\sigma)}{\sigma^{2}}[\delta(1-\sigma)-\rho]+(1-\alpha) \delta \frac{\delta(\sigma-\alpha)+\alpha \rho}{2 \alpha^{2} \sigma^{2}}(2 \alpha-\sigma)$

where $G(\sigma)=-B(\mathbf{J}) \cdot \operatorname{tr}(\mathbf{J})+\operatorname{Det}(\mathbf{J})$, which vanishes at different solutions of $\sigma$ (see, Fig. 1).

Example 1 Let $(\rho, \alpha, \delta)=(0.05,0.33,0.25)$ which are standard values in the literature. Therefore, $G(\sigma)=0$ when $\bar{\sigma}_{1}=0.4538$ and $\bar{\sigma}_{2}=0.6368$.

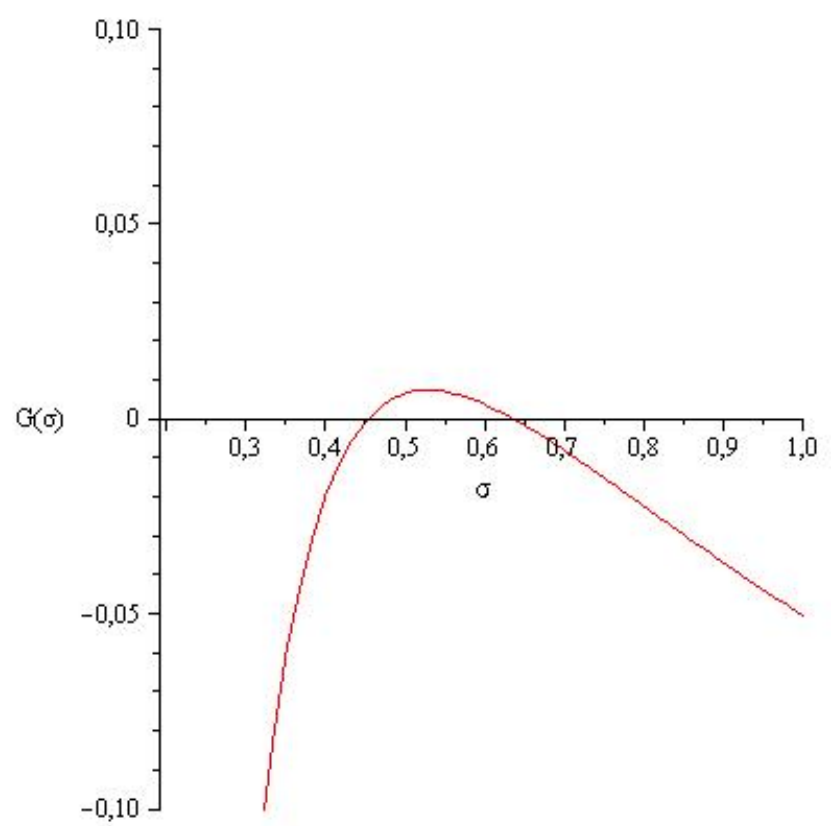

Fig. 1: The Hopf bifurcation curve

Multiple values of $\sigma$ are thus able to annihilate $G(\sigma)$. If this happens, we will show that any variation of $\sigma$ around $\bar{\sigma}_{i}, i \in[1,2]$ can force the variables 
associated to the complex conjugate eigenvalues to oscillate around a common constant value. This means also that, an invariant cycle (a closed orbit) may emerge around the steady state, or collapses onto it. We want to show that parameter $\sigma$ plays a crucial role in the characterization of an optimal solution to our maximization problem, and thus matters in the process of a long run sustainable growth, giving rise to a boom and bust sustained cycle, where tourism services lead growth but start exploiting natural resources until economic indicators start a sharp and rapid contraction to different places, thus lowering human impact on the environment, which starts restoring natural capital and thus enters a new phase of tourism attracting place, which pushes up again the economic activities in a periodic evolution consistent with the life-cycle hypothesis.

Unfortunately, this analysis may not be sufficient to provide a complete picture of the stability properties of the economy, for more complicated outcomes can emerge if we move slightly off the vicinity of the steady state, and may thus complicate the adequate policy actions to be implemented. A deep investigation of this issue is provided in the next section.

\section{Periodic orbits and global indeterminacy}

Studying the properties of an equilibrium outside the small neighborhood of the steady state is not an easy task, especially when dealing with non-linear functions that complicate the algebraic calculations behind it.

Firstly, we need to put the system $(R)$ in an appropriate canonical form to work with. To do this, we translate the equilibrium fixed point to the origin, by assuming

$$
\begin{aligned}
& \tilde{x}=x-x^{*}, \\
& \tilde{q}=q-q^{*} \\
& \tilde{z}=z-z^{*}
\end{aligned}
$$

which implies when resources are depleted and mass tourism moves

$$
\begin{aligned}
& \dot{x}=\left\{-\frac{\rho}{\sigma}+\left(\frac{\alpha-\sigma}{\sigma}\right) A\left(q^{*}+\tilde{q}\right)^{\alpha-1}\left(z^{*}+\tilde{z}\right)^{(1-\alpha)}+\left(x^{*}+\tilde{x}\right)\right\}\left(x^{*}+\tilde{x}\right) \\
& \dot{q}=\left\{-\delta+A\left(q^{*}+\tilde{q}\right)^{\alpha-1}\left(z^{*}+\tilde{z}\right)^{(1-\alpha)}-\left(x^{*}+\tilde{x}\right)+\delta\left(z^{*}+\tilde{z}\right)\right\}\left(q^{*}+\tilde{q}\right) \\
& \dot{z}=\left\{\delta \frac{1-\alpha}{\alpha}-\left(x^{*}+\tilde{x}\right)+\delta\left(z^{*}+\tilde{z}\right)\right\}\left(z^{*}+\tilde{z}\right)
\end{aligned}
$$

A second order Taylor expansion of this vector field allows us to put $(Q)$ in the form

$$
\left(\begin{array}{c}
\dot{x} \\
\dot{q} \\
\dot{z}
\end{array}\right)=\mathbf{J}\left(\begin{array}{c}
\tilde{x} \\
\tilde{q} \\
\tilde{z}
\end{array}\right)+\left(\begin{array}{c}
\tilde{f}_{1}(\tilde{x}, \tilde{q}, \tilde{z}) \\
\tilde{f}_{2}(\tilde{x}, \tilde{q}, \tilde{z}) \\
\tilde{f}_{3}(\tilde{x}, \tilde{q}, \tilde{z})
\end{array}\right)
$$

where the $\tilde{f}_{i}$ terms represent the non linear parts (of order 2).

Proposition 2 Let $\mathbf{T}$ be a matrix of the eigenvectors associated with the structure of eigenvalues of $\mathbf{J}$ at the bifurcation point. Then, it is possible to put the system (Q) in the following Jordan normal form:

$$
\dot{\mathbf{w}}=\mathbf{T}^{-1} \mathbf{J}(0) \mathbf{T} \mathbf{w}+F
$$

where $F=\mathbf{T}^{-1} \tilde{f}_{i}(\mathbf{T w})$, given the associated change in coordinates

$$
\left(\begin{array}{l}
\tilde{x} \\
\tilde{q} \\
\tilde{z}
\end{array}\right)=\mathbf{T}\left(\begin{array}{l}
w_{1} \\
w_{2} \\
w_{3}
\end{array}\right)
$$

which transforms system $(Q)$ into

$$
\left(\begin{array}{l}
\dot{w}_{1} \\
\dot{w}_{2} \\
\dot{w}_{3}
\end{array}\right)=\left[\begin{array}{lll}
0 & 1 & 0 \\
0 & 0 & 1 \\
\varepsilon_{1} & \varepsilon_{2} & \varepsilon_{3}
\end{array}\right]\left(\begin{array}{l}
w_{1} \\
w_{2} \\
w_{3}
\end{array}\right)+\left(\begin{array}{l}
F_{1}\left(w_{1}, w_{2}, w_{3}\right) \\
F_{2}\left(w_{1}, w_{2}, w_{3}\right) \\
F_{3}\left(w_{1}, w_{2}, w_{3}\right)
\end{array}\right)
$$

where $\varepsilon_{1}=\operatorname{Det}(\mathbf{J}), \varepsilon_{2}=-B(\mathbf{J}), \varepsilon_{3}=\operatorname{tr}(\mathbf{J})$, and $F_{i}$ are the transformed second order non linear terms.

Proof. See the procedure detailed in Algaba et al. (1999) and Gamero et al. (1991). 
We are thus able at this step to restrict the vector field in (10) to the plane $\left(w_{1}, w_{2}\right)$ whose eigenspace, at the bifurcation value $\sigma=\bar{\sigma}$, corresponds to the complex pair of eigenvalues, $\lambda_{1,2}= \pm \omega i$, which is topologically invariant with respect to the original system $(S)$. That is, if we substitute $B J \cdot t r J=D e t J$ in the characteristic equation at the bifurcation point (i.e., $G(\bar{\sigma})=0$ ), one eigenvalue is real and positive, and equal to the trace, $\lambda_{1}=\operatorname{tr} J$, whilst the other two eigenvalues are complex conjugate, $\lambda_{2,3}= \pm \omega i$, assuming $\omega=\sqrt{B J}$.

A center manifold reduction of the linearized vector field allows us to investigate this case.

Proposition $3 A$ second order approximation of the center manifold which reduces the vector field in (10) is given by the following equation $w_{3}=h\left(w_{1}, w_{2}\right)=\frac{1}{2}\left[\tau_{1} w_{1}^{2}+\tau_{2} w_{1} w_{2}+\tau_{3} w_{2}^{2}\right]$, where $\tau_{i}$ are coefficients that satisfy the stability condition $\dot{w}_{3}=0$.

Proof. See Appendix.

The vector field at the center manifold therefore reduces to

$\left(\begin{array}{c}\dot{w}_{1} \\ \dot{w}_{2}\end{array}\right)=\left[\begin{array}{cc}0 & -\omega \\ \omega & 0\end{array}\right]\left(\begin{array}{l}w_{1} \\ w_{2}\end{array}\right)+\left(\begin{array}{l}\bar{F}^{1}\left(w_{1}, w_{2}, h\left(w_{1}, w_{2}\right)\right) \\ \bar{F}^{2}\left(w_{1}, w_{2}, h\left(w_{1}, w_{2}\right)\right)\end{array}\right)$

where $\bar{F}^{i}$ represent the second order non linear terms of the vector field at the center manifold.

The restricted vector field (11) allows us to properly investigate the presence of periodic solutions in the two-dimensional phase space $\left(w_{1}, w_{2}\right)$, by computing the standard first Lyapunov coefficient

$q=\frac{1}{16 \omega}\left[\bar{F}_{w_{1} w_{2}}^{1}\left(\bar{F}_{w_{2} w_{2}}^{1}+\bar{F}_{w_{1} w_{1}}^{1}\right)-\bar{F}_{w_{1} w_{2}}^{2}\left(\bar{F}_{w_{2} w_{2}}^{2}+\bar{F}_{w_{1} w_{1}}^{2}\right)-\bar{F}_{w_{1}}^{1}\right.$
Remark 2 If $q<0$ the emerging cycle around the steady state is attracting, i.e. a supercritical Hopf bifurcation occurs. (The statement is reversed for $q>0$.)

The value of $q$, at the two bifurcation points, can be either positive or negative. Both bifurcations can therefore be supercritical or subcritical. The fixed points are thus unstable and the orbits are attracting on the center manifold. This is shown by means of the following numerical example.

Example 2 Assume $\rho=0.05, \quad \alpha=0.33$, $\delta=0.25$ as in Example 1. If $\sigma=\bar{\sigma}_{1}$, then $q=-8.8 \cdot 10^{-10}<0$, that is to say the bifurcation is supercritical, the steady state is unstable and the periodic orbits are attracting on the center manifold. On the contrary, in correspondence of $\sigma=\bar{\sigma}_{2}$, we have $q=2.54 \cdot 10^{14}>0$, that is to say the bifurcation is subcritical, and the periodic orbits start repelling (see, Fig. 2).

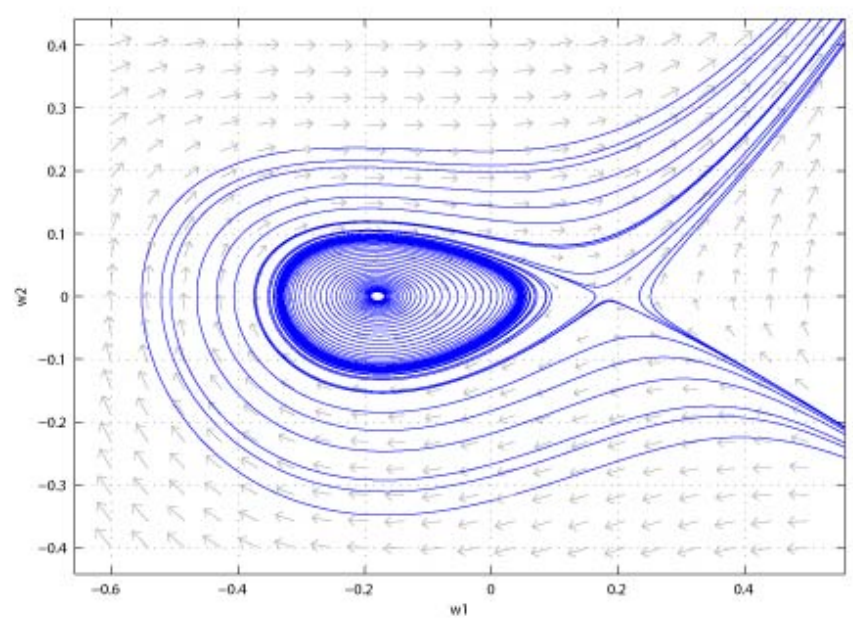

Fig. 2: Periodic orbits near the equilibrium

We are thus able to conclude that different periodic solutions may emerge in presence of resource

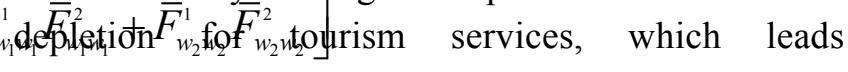
consequently to the rise of some indeterminacy problems, which might be able to explain the rise and fall of different nowadays tourism-based economies that, even though endowed with the same initial conditions, may at some point start to perform 
differently in growth rate terms, and thus follow different long run equilibrium paths.

\section{Concluding remarks}

The raise of multiple equilibria and indeterminacy of the steady state solution has been commonly investigated in the literature to explain the diversity of growth rates across countries. However, when the system is characterized by highly nonlinear relationships the resulting dynamics around the steady state can be even more complex.

To shed some light in this field, we presented a model, arguing that a crucial aspect for the occurrence of both indeterminacy and cyclical adjustment towards the steady state might be the presence of particular bifurcation values of the inverse of the intertemporal elasticity of substitution. Conclusions to our analysis confirm that such parameter matters in the transition towards a long-run sustainable equilibrium, thus leaving space to other more complicated dynamic phenomena characterized by periodic solutions that stuck the economy in a low level equilibrium trap.

\section{References}

Algaba, A., Freire, E., \& Gamero, E. (1999). Hypernormal forms for equilibria of vector fields. Codimension one linear degeneracies. Rocky Mountain Journal of Mathematics, 29, 13-45.

Baggio, R. (2008). Symptoms of complexity in a tourism system. Tourism Analysis, 13(1), 1-20.

Bella, G., \& Mattana, P. (2014). Global indeterminacy of the equilibrium in the Chamley model of endogenous growth in the vicinity of a Bogdanov-Takens bifurcation. Mathematical Social Sciences, 71, 69-79.

Benhabib, J., Schmitt-Grohé, S., \& Uribe, M. (2001). The perils of Taylor rules. Journal of Economic Theory, 96, 40-69.

Bornhorst, T., Ritchie, J., \& Sheehan, L. (2010). Determinants of Tourism Success for DMOs \& destinations: An Empirical Examination of Stakeholders' Perspectives. Tourism Management, 31, 572-589.

Brau, R., Lanza, A., \& Pigliaru, F. (2007). How fast are small tourism countries growing? Evidence from the data for 1980-2003. Tourism Economics, 13(4), 603613
Brida, J. G., Sanchez Carrera, E. J., \& Risso, W. A. (2008). Tourism's Impact on Long-Run Mexican Economic Growth. Economics Bulletin, 3(21), 1-8.

Butler, R. (1980). The concept of a tourist area cycle of evolution. Canadian Geographer, 24, 5-12.

Cole, S. (2009). A logistic tourism model: Resort Cycles, Globalization and Chaos. Annals of Tourism Research, 36(4), 689-714.

Finco, M. V. A. (2009). Poverty-Environment Trap: A non linear probit model applied to rural areas in the North of Brazil. American-Eurasian J. Agric. \& Environ. Sci., 5(4), 533-539.

Gamero, E., Freire, E., \& Ponce, E. (1991). Normal forms for planar systems with nilpotent linear part. In R. Seydel, F. W. Schneider, T. Küpper, \& H. Troger (Eds.), Bifurcation and Chaos: Analysis, Algorithms, Applications. International Series of Numerical Mathematics, (pp. 123-12). Basel, Birkhäuser.

Katircioglu, S. T. (2009). Testing the tourism-led growth hypothesis: The case of Malta. Acta Oeconomica, 59(3), 331-343.

Mattana, P., \& Venturi, B. (1999). Existence and stability of periodic solutions in the dynamics of endogenous growth. International Review of Economics and Business, 46, 259-284.

Musu, I. (1995). Transitional Dynamics to Optimal Sustainable Growth. FEEM Working Paper 50.95.

Nowak, J. J., Sahli, M., \& Cortés-Jiménez, I. (2007). Tourism, capital good imports and economic growth: theory and evidence for Spain. Tourism Economics, 13(4), 515-536.

Rosendahl, K. E. (1996). Does improved environmental policy enhance economic growth?. Environmental and Resource Economics, 9, 341-364.

Sachs, J. D., \& Warner, A. M. (2001). Natural resources and economic development: The curse of natural resources. European Economic Review, 45, 827-838.

Schubert, F. S., Brida, J. G., \& Risso, W. A. (2010). The impacts of international Tourism demand on economic growth of small economies dependent of tourism. Tourism Management, 32(2), 377-385.

Shang, D., \& Han, M. (2005). The existence of homoclinic orbits to saddle-focus. Applied Mathematics and Computation, 163, 621-631.

Shilnikov, L. P. (1965). A case of the existence of a denumerate set of periodic motions. Sov. Math. Docl., $6,163-166$. 


\section{Appendix}

The Current value Hamiltonian associated with system $P$ is given by:

$$
\begin{array}{ll}
H_{C}=\frac{c^{1-\sigma}-1}{1-\sigma}+\lambda\left[A k^{\alpha} g^{\beta(1-\alpha)} E^{(1-\beta)(1-\alpha)}-c\right]+\mu\left[\delta\left(E-g^{\beta} E^{1-\beta}\right)\right. & \frac{\dot{q}}{q}=\left(\frac{\dot{k}}{k}-\frac{\dot{E}}{E}\right)=-\delta+A q^{\alpha-1} z^{(1-\alpha)}-x+\delta z \\
\text { where } \lambda \text { and } \mu \text { represent the shadow prices of } & \frac{\dot{z}}{z}=\beta\left(\frac{\dot{g}}{g}-\frac{\dot{E}}{E}\right)=\delta \frac{1-\alpha}{\alpha}-x+\delta z
\end{array}
$$
physical and natural capital, respectively. The first order condition for a maximum requires that the discounted Hamiltonian be maximized with respect to its control variables, which implies

$$
\begin{aligned}
& c^{-\sigma}=\lambda \quad \text { (A.1) } \\
& \lambda(1-\alpha) A k^{\alpha}=\mu \delta g^{\beta \alpha} E^{(1-\beta) \alpha}
\end{aligned}
$$

accompanied by the law of motion of each costate variable

$$
\begin{aligned}
& \frac{\dot{\lambda}}{\lambda}=\rho-\alpha A k^{\alpha-1} g^{\beta(1-\alpha)} E^{(1-\beta)(1-\alpha)} \\
& \frac{\dot{\mu}}{\mu}=\rho-(1-\beta) \frac{\lambda}{\mu}(1-\alpha) A k^{\alpha} g^{\beta(1-\alpha)} E^{(1-\beta)(1-\alpha)-1}-\delta
\end{aligned}
$$

(A.4)

Taking log-derivatives of (A.1) and (A.2), we derive:

$$
\begin{aligned}
& -\sigma \frac{\dot{c}}{c}=\frac{\dot{\lambda}}{\lambda} \\
& \frac{\dot{\lambda}}{\lambda}+\alpha \frac{\dot{k}}{k}-\beta \alpha \frac{\dot{g}}{g}-(1-\beta) \alpha \frac{\dot{E}}{E}=\frac{\dot{\mu}}{\mu}
\end{aligned}
$$

Therefore, problem $(P)$ can be defined by the following system of four first order differential equations:

$$
\begin{aligned}
& \frac{\dot{k}}{k}=A k^{\alpha-1} g^{\beta(1-\alpha)} E^{(1-\beta)(1-\alpha)}-\frac{c}{k} \\
& \frac{\dot{c}}{c}=-\frac{\rho}{\sigma}+\frac{\alpha}{\sigma} A k^{\alpha-1} g^{\beta(1-\alpha)} E^{(1-\beta)(1-\alpha)} \\
& \frac{\dot{E}}{E}=\delta\left(1-g^{\beta} E^{-\beta}\right) \\
& \frac{\dot{g}}{g}=-\frac{1}{\beta} \frac{c}{k}+\frac{\delta(1-\alpha+\alpha \beta)}{\beta \alpha}+\frac{\delta}{\beta}(1-\beta) g^{\beta} E^{-\beta}
\end{aligned}
$$

To ease the analysis, system (A.7) can be further reduced, by the following convenient variable substitution, $x=\frac{c}{k}, q=\frac{k}{E}$, and $z=\left(\frac{g}{E}\right)^{\beta}$. That is to say,
(A.8)

with the associated steady state values

$$
\begin{aligned}
& x^{*}=\frac{\delta(\sigma-\alpha)+\alpha \rho}{\alpha \sigma} \\
& q^{*}=\left[\frac{\rho-\delta(1-\sigma)}{\delta \sigma}\right]\left(\frac{A \alpha}{\delta}\right)^{1-\alpha} \\
& z^{*}=\frac{\rho-\delta(1-\sigma)}{\delta \sigma}
\end{aligned}
$$

The Jacobian matrix of the reduced system (A.9) is then

$$
\left[\begin{array}{ccc}
\mathbf{J}=\left(1-\beta^{\gamma}\right)^{*} g^{\beta} & \frac{\delta(\alpha-1)}{E^{-\rho} \frac{P}{\alpha}}\left(\frac{\alpha-\sigma}{\sigma}\right) \frac{x^{*}}{q^{*}} & \frac{\delta(1-\alpha)}{\alpha}\left(\frac{\alpha-\sigma}{\sigma}\right) \frac{x^{*}}{z^{*}} \\
q^{*} & \frac{\delta}{\alpha}(\alpha-1) & \delta q^{*}+\frac{\delta(1-\alpha)}{\alpha} \frac{q^{*}}{z^{*}} \\
-z^{*} & 0 & \delta z^{*}
\end{array}\right]
$$

with the associated Trace, Determinant and Sum of Principal Minors, respectively given by:

$$
\begin{aligned}
& \operatorname{trJ}=2 \delta z^{*} \quad(\mathrm{~A} .10) \\
& \operatorname{Det} J=\frac{\delta^{2}(1-\alpha)(2 \alpha-3 \sigma)}{\alpha \sigma} x^{*} z^{*} \\
& B J=\delta z^{*} x^{*}-(1-\alpha) \frac{\delta}{\alpha}\left[x^{*}+\delta z^{*}\right]
\end{aligned}
$$

Translation to the origin.

Substitute $\tilde{x} \equiv x-x^{*}, \quad \tilde{q} \equiv q-q^{*}, \quad \tilde{z}=z-z^{*}$ in the reduced system (A.8).

$$
\begin{aligned}
& \dot{x}=\left\{-\frac{\rho}{\sigma}+\left(\frac{\alpha-\sigma}{\sigma}\right) A\left(q^{*}+\tilde{q}\right)^{\alpha-1}\left(z^{*}+\tilde{z}\right)^{(1-\alpha)}+\left(x^{*}+\tilde{x}\right)\right\}\left(x^{*}+\tilde{x}\right) \\
& \dot{q}=\left\{-\delta+A\left(q^{*}+\tilde{q}\right)^{\alpha-1}\left(z^{*}+\tilde{z}\right)^{(1-\alpha)}-\left(x^{*}+\tilde{x}\right)+\delta\left(z^{*}+\tilde{z}\right)\right\}\left(q^{*}+\tilde{q}\right) \\
& \dot{z}=\left\{\delta \frac{1-\alpha}{\alpha}-\left(x^{*}+\tilde{x}\right)+\delta\left(z^{*}+\tilde{z}\right)\right\}\left(z^{*}+\tilde{z}\right)
\end{aligned}
$$

A second order Taylor expansion of (A.13) can be computed: 


$$
\left(\begin{array}{c}
\dot{x} \\
\dot{q} \\
\dot{z}
\end{array}\right)=\mathbf{J}\left(\begin{array}{c}
\tilde{x} \\
\tilde{q} \\
\tilde{z}
\end{array}\right)+\left(\begin{array}{c}
\tilde{f}_{1}(\tilde{x}, \tilde{q}, \tilde{z}) \\
\tilde{f}_{2}(\tilde{x}, \tilde{q}, \tilde{z}) \\
\tilde{f}_{3}(\tilde{x}, \tilde{q}, \tilde{z})
\end{array}\right)
$$

being $\tilde{f}_{i}$ the nonlinear terms of the expanded vector field:

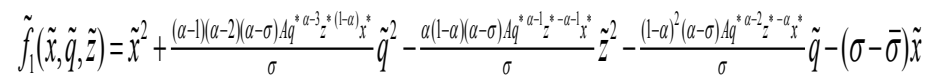

$$
\tilde{f}_{2}(\tilde{x}, \tilde{q}, \tilde{z})=(\alpha-1)^{2} A q^{* \alpha-2} z^{*(1-\alpha)} \tilde{q}^{2}-\alpha(1-\alpha) A q^{* \alpha-1} z^{*-a n d} q^{*} \tilde{z}^{2}-\tilde{x} \tilde{q}-(1-\alpha)^{2} A q^{* \alpha-1} z^{*-\alpha} \tilde{q} \tilde{z}
$$

$$
\tilde{f}_{3}(\tilde{x}, \tilde{q}, \tilde{z})=\delta \tilde{z}^{2}-\tilde{x} \tilde{z}
$$

Following the detailed procedure in Algaba et al. (2003), system (A.14) can be put in a more convenient Jordan normal form,

$$
\left(\begin{array}{l}
\dot{w}_{1} \\
\dot{w}_{2} \\
\dot{w}_{3}
\end{array}\right)=\left[\begin{array}{lll}
0 & 1 & 0 \\
0 & 0 & 1 \\
\varepsilon_{1} & \varepsilon_{2} & \varepsilon_{3}
\end{array}\right]\left(\begin{array}{l}
w_{1} \\
w_{2} \\
w_{3}
\end{array}\right)+\left(\begin{array}{l}
F_{1}\left(w_{1}, w_{2}, w_{3}\right) \\
F_{2}\left(w_{1}, w_{2}, w_{3}\right) \\
F_{3}\left(w_{1}, w_{2}, w_{3,}\right)
\end{array}\right)
$$

(A.15)

via the change of coordinates

$$
\left(\begin{array}{l}
\tilde{x} \\
\tilde{q} \\
\tilde{z}
\end{array}\right)=\mathbf{T}\left(\begin{array}{l}
w_{1} \\
w_{2} \\
w_{3}
\end{array}\right)
$$

made by the transformation matrix $\mathbf{T}=[\mathbf{u}, \mathbf{v}, \mathbf{z}]^{T}$ with

$$
\mathbf{u}=\left[\begin{array}{c}
-\frac{j_{23}^{*}}{j_{31}^{*}} \\
\frac{j_{11}^{*} j_{23}^{*}-j_{13}^{*} j_{31}^{*}}{j_{12}^{*} j_{31}^{*}} \\
1
\end{array}\right], \mathbf{v}=\left[\begin{array}{c}
-\frac{1}{z} \\
\frac{x+\delta q+(1-\alpha) A q^{\alpha} z^{-\alpha}}{q z} \\
0
\end{array}\right] \mathbf{z}=\left[\begin{array}{c}
-\frac{j_{33}^{*}}{j_{31}^{*}} \\
0 \\
1
\end{array}\right]
$$

and where $\varepsilon_{1}=\operatorname{Det} J, \varepsilon_{2}=-B J, \varepsilon_{3}=\operatorname{Tr} J$, being $F_{i}=\mathbf{T}^{-1} \tilde{f}_{i}(\mathbf{T w})$ the transformed second order non linear terms. To study the stability of periodic orbits around the steady state, we consider the Andronov-Hopf bifurcation coefficient: $q=\frac{1}{16 \omega}\left[\bar{F}_{w_{1} w_{2}}^{1}\left(\bar{F}_{w_{2} w_{2}}^{1}+\bar{F}_{w_{1} w_{1}}^{1}\right)-\bar{F}_{w_{1} w_{2}}^{2}\left(\bar{F}_{w_{2} w_{2}}^{2}+\bar{F}_{w_{1} w_{1}}^{2}\right)-\bar{F}_{w_{1} w_{1}}^{1} \bar{F}_{w_{1} w_{1}}^{2}+\bar{F}_{w_{2} w_{2}}^{1} \bar{F}_{w_{2} w_{2}}^{2}\right]$

where

$$
\begin{aligned}
\bar{F}_{w_{2} w_{2}}^{2}= & 2(\alpha-1)^{2} A \bar{q}^{* \alpha-2} \bar{z}^{*(1-\alpha)} v_{2}-2 v_{1} v_{2} \\
\bar{F}_{w_{1} w_{1}}^{2}= & 2(\alpha-1)^{2} A \bar{q}^{* \alpha-2} \bar{z}^{*(1-\alpha)} u_{2}-2 \alpha(1-\alpha) A \bar{q}^{* \alpha-1} \bar{z}^{*-\alpha-1} \bar{q}^{*}- \\
& -2 u_{1} u_{2}-2(1-\alpha)^{2} A \bar{q}^{* \alpha-1} \bar{z}^{*-\alpha} u_{2}
\end{aligned}
$$

\section{Computation of the center manifold.}

Assume $w_{3}=h\left(w_{1}, w_{2}\right)$ with $h$ smooth function. The properties of the center manifold imply

$$
\dot{w}_{3}-\frac{\partial h}{\partial w_{1}} \dot{w}_{1}-\frac{\partial h}{\partial w_{2}} \dot{w}_{2}=0
$$

\section{Suppose now}

$$
w_{3}=h\left(w_{1}, w_{2}\right)=\frac{1}{2}\left[\tau_{1} w_{1}^{2}+\tau_{2} w_{1} w_{2}+\tau_{3} w_{2}^{2}\right]
$$

where $\tau_{1}, \tau_{2}$ and $\tau_{3}$ are unknown coefficients. Time-differentiating (A.17), and substituting into (A.16), we obtain the following relation

$$
w_{1}^{2}+\frac{\Phi \operatorname{Tr} J \tau_{2}+2 v_{2} A_{3}^{\prime}-2 v_{1} B_{3}^{\prime}+2 \chi C_{3}^{\prime}-2 \Phi \tau_{1}}{\Phi T r J \tau_{1}+2 v_{2} A_{1}^{\prime}-2 v_{1} B_{1}^{\prime}+2 \chi C_{1}^{\prime}} w_{1} w_{2}+\frac{\Phi T r J \tau_{3}+2 v_{2} A_{2}^{\prime}-2 v_{1} B_{2}^{\prime}+2 \chi C_{2}^{\prime}-\Phi \tau_{2}}{\Phi \operatorname{Tr} J \tau_{1}+2 v_{2} A_{1}^{\prime}-2 v_{1} B_{1}^{\prime}+2 \chi C_{1}^{\prime}} w_{2}^{2}=0
$$

Finally, equating coefficients to zero, we find

$$
\begin{aligned}
& \tau_{1}=\frac{2\left[-v_{2} A_{1}^{\prime}+v_{1} B_{1}^{\prime}-\chi C_{1}^{\prime}\right]}{\Phi T r J} ; \quad \tau_{2}=\frac{2 \operatorname{Tr} J\left[-v_{2} A_{3}^{\prime}+v_{1} B_{3}^{\prime}-\chi C_{3}^{\prime}\right]+4\left(\left[-v_{2} A_{1}^{\prime}+v_{1} B_{1}^{\prime}-\chi C_{1}^{\prime}\right]\right)}{\Phi T r J^{2}} \\
& \tau_{3}=\frac{2\left[-v_{2} A_{2}^{\prime}+v_{1} B_{2}^{\prime}-\chi C_{2}^{\prime}\right] \operatorname{Tr} J^{2}+2 T r J v+4\left[-v_{2} A_{1}^{\prime}+v_{1} B_{1}^{\prime}-\chi C_{1}^{\prime}\right]}{\Phi T r J^{3}} \\
& \text { where all other coefficients are intricated }
\end{aligned}
$$
Combinations of the nonlinear terms $\bar{F}_{i}$, which are not reported for the sake of simplicity, but remain available upon request. 